\title{
A novel carotenoid 1,2-hydratase (CruF) from two species of the non-photosynthetic bacterium Deinococcus
}

\author{
Correspondence \\ Bing Tian \\ tianbing@zju.edu.cn \\ or \\ Yuejin Hua \\ yjhua@zju.edu.cn
}

Received 18 January 2009

Revised 29 April 2009

Accepted 8 May 2009

\section{Zongtao Sun, ${ }^{1}$ Shaochuan Shen, ${ }^{1,2}$ Chao Wang, ${ }^{1}$ Hu Wang, ${ }^{1}$ Yaping $\mathrm{Hu},{ }^{1}$ Jiandong Jiao, ${ }^{1}$ Tingting Ma, ${ }^{1}$ Bing $\operatorname{Tian}^{1}$ and Yuejin Hua ${ }^{1}$}
${ }^{1}$ Key Laboratory for Nuclear-Agricultural Sciences of Chinese Ministry of Agriculture and Zhejiang Province, Institute of Nuclear-Agricultural Sciences, Zhejiang University, 310029 Hangzhou, China
${ }^{2}$ State Key Laboratory Breeding Base of Green Chemistry Synthesis Technology, College of Chemical Engineering and Materials Science, Zhejiang University of Technology, 310032 Hangzhou, China

\begin{abstract}
A novel carotenoid 1,2-hydratase (CruF) responsible for the $\mathrm{C}-1^{\prime}, 2^{\prime}$ hydration of $\gamma$-carotene was identified in the non-photosynthetic bacteria Deinococcus radiodurans $\mathrm{R} 1$ and Deinococcus geothermalis DSM 11300. Gene expression and disruption experiments demonstrated that dr0091 and dgeo2309 encode CruF in D. radiodurans and D. geothermalis, respectively. Their homologues were also found in the genomes of cyanobacteria, and exhibited little homology to the hydroxyneurosporene synthase $(\mathrm{CrtC})$ proteins found mainly in photosynthetic bacteria. Phylogenetic analysis showed that CruF homologues form a separate family, which is evolutionarily distant from the known CrtC family.
\end{abstract}

\section{INTRODUCTION}

Carotenoids, natural antioxidative pigments important to human health, are usually synthesized in bacteria, algae, fungi and plants, and typically consist of $\mathrm{C}_{40}$ hydrocarbon backbones (carotenes) and their oxygenated derivatives (xanthophylls) (Armstrong, 1997). Biosynthesis of $\mathrm{C}_{40}$ carotenoid begins at the isoprenoid or terpenoid pathway. The head-to-head condensation of two geranylgeranyl disphosphate (GGPP) molecules forms a colourless carotenoid phytoene, catalysed by phytoene synthase (CrtB). Phytoene is then converted to lycopene by phytoene desaturase (CrtI), or by phytoene desaturase (CrtP), $\zeta$-carotene desaturase (CrtQ) and cis-carotene isomerase (CrtH) (Takaichi \& Mochimaru, 2007). Following desaturation, carotenoid biosynthesis is diversified into acyclic and cyclic carotenoids. For cyclization, one or both $\psi$-end groups of lycopene are cyclized into $\gamma$ carotene or $\beta$-carotene by lycopene cyclase. For further modification of acyclic and monocyclic carotenoids, the $\psi$ end group can be hydrated by carotenoid 1,2-hydratase at C-1 (or 1') and C-2 (or 2') (Schmidt-Dannert, 2000).

Species of the genus Deinococcus are red-pigmented nonphotosynthetic bacteria, well known for their extreme tolerance to ionizing radiation and numerous oxidative

Abbreviations: GGPP, geranylgeranyl disphosphate; ROS, reactive oxygen species.

A supplementary table of primers and two supplementary figures are available with the online version of this paper. damaging agents (Cox \& Battista, 2005; Ferreira et al., 1997). D. radiodurans produces deinoxanthin, a unique hydroxylated carotenoid, as its major carotenoid (Lemee et al., 1997; Saito et al., 1998). Deinoxanthin was shown to have stronger reactive oxygen species (ROS) scavenging ability than lycopene and $\beta$-carotene, due to its extended conjugated double bonds and functional end groups, such as hydroxyl groups (Tian et al., 2007). We have previously identified phytoene synthase (CrtB, DR0862), phytoene desaturase (CrtI, DR0861) and carotenoid 3',4' -desaturase (CrtD, DR2250), which are involved in steps of deinoxanthin biosynthesis (Tian et al., 2007, 2008; Xu et al., 2007). Zhang et al. (2007) also confirmed the functions of the $\operatorname{crtB}$ and crtI genes. Two other genes, encoding carotenoid ketolase (CrtO) and lycopene cyclase ( $\mathrm{CrtLm})$, in D. radiodurans have been described (Tao \& Cheng, 2004; Tao et al., 2004). Carotenoid 1,2-hydratase, a carotenoid biosynthetic enzyme, is required to catalyse the synthesis of deinoxanthin by hydration at the $\mathrm{C}-1^{\prime}, 2^{\prime}$ double bond. CrtC-type carotenoid 1,2-hydratases have been found in photosynthetic bacteria (Armstrong et al., 1989; Frigaard et al., 2004; Kovács et al., 2003; Ouchane et al., 1997), primarily involved in the biosynthesis of spirilloxanthin, spheroidene, chlorobactene and its derivatives. However, no homologue of the CrtC-type carotenoid 1,2-hydratase has been detected in the $D$. radiodurans genome (Makarova et al., 2001; White et al., 1999). We identified the gene candidates of carotenoid 1,2-hydratase, dr0091 and dgeo2309, predicted in the gene neighbourhood of $\mathrm{crtO}$ 
from $D$. radiodurans $\mathrm{R} 1$ and $D$. geothermalis DSM 11300, respectively. The homologue of $d r 0091$ and dgeo2309 from the cyanobacterium Synechococcus sp. PCC 7002 was recently described and named CruF by Maresca et al. (2008).

In this study, we identified and characterized a CruF-type carotenoid 1,2-hydratase from $D$. radiodurans $\mathrm{R} 1$ and $D$. geothermalis DSM 11300. The results helped to reveal the biosynthetic pathway of deinoxanthin in $D$. radiodurans (Fig. 1). The CruF homologues form a separate family of carotenoid 1,2-hydratase, and are evolutionarily distant from the known hydroxyneurosporene synthase ( $\mathrm{CrtC}$ ) family.

\section{METHODS}

Bacterial strains and growth conditions. All strains and plasmids used in this study are listed in Table 1 . The wild-type and mutant strains of D. radiodurans R1 (ATCC 13939) were grown at $30{ }^{\circ} \mathrm{C}$ and the wild-type strain of $D$. geothermalis DSM 11300 was grown at $50{ }^{\circ} \mathrm{C}$ in TGY medium $[0.5 \%(\mathrm{w} / \mathrm{v})$ Bacto tryptone, $0.1 \%(\mathrm{w} / \mathrm{v})$ glucose, and $0.3 \%(\mathrm{w} / \mathrm{v})$ Bacto yeast extract] on an orbital shaker or on TGY plates solidified with $1.5 \%(w / v)$ agar. Escherichia coli was cultured in LB medium at $37^{\circ} \mathrm{C}$. Tetracycline $\left(25 \mu \mathrm{g} \mathrm{ml} \mathrm{ml}^{-1}\right)$, ampicillin $\left(60 \mu \mathrm{g} \mathrm{ml}^{-1}\right)$, chloramphenicol $\left(30 \mu \mathrm{g} \mathrm{ml}^{-1}\right)$ or kanamy$\operatorname{cin}\left(20 \mu \mathrm{g} \mathrm{ml}^{-1}\right)$ was added to the medium if required.

Sequence and phylogenetic analysis. The gene neighbourhoods of $c r t O$ homologues in the genomes of $D$. radiodurans $\mathrm{R} 1$ and $D$. geothermalis DSM 11300 (http://ncbi.nlm.nih.gov) were inspected for gene candidates encoding carotenoid biosynthetic enzymes. Two nearby sequences (ORFs), dr0091 and dgeo2309, were selected for functional analysis. Their sequences were used to retrieve the homologues in other organisms by the BLAST program (http:// blast.ncbi.nlm.nih.gov/Blast.cgi). The known CrtC protein sequences were obtained from the Swiss-Prot database (http://www.expasy.org/). Multiple sequences were aligned using the CLUSTAL W program (http://www.ebi.ac.uk/clustalw/). The evolutionary tree of protein sequences was constructed by the neighbour-joining (NJ) method with protein p-distances by the MEGA3.1 (Molecular Evolutionary Genetics Analysis) software (http://www.megasoftware.net/ index.html), and the reliability of the tree topology was assessed by 1000 bootstrap replications.

Construction of expression plasmids. The dr0091 or dgeo2309 gene was amplified from $D$. radiodurans and D. geothermalis by the primers RKdr91 P1 and RKdr91 P2 or the primers RKdg2309 P1 and RKdg2309 P2, respectively (primer sequences are listed in Supplementary Table S1, available with the online version of this paper). The PCR products were ligated into TA cloning vector pMD18-T. The HindIII/BamHI-digested fragments were cloned in the HindIII/BamHI site of pRK404, yielding pRK-DR91 or pRKDG2309 (Table 1).

E. coli transformants containing pRK-DR91 or pRK-DG2309 as well

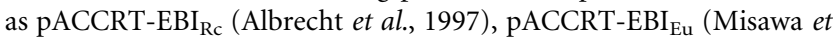
al., 1995) or pAC-GAMMA (Cunningham \& Gantt, 2005) were incubated at $28{ }^{\circ} \mathrm{C}$. IPTG was added to a final concentration of $0.5 \mathrm{mM}$ when the $\mathrm{OD}_{600}$ of the culture reached about 0.5 .

Construction of mutant strains. Mutants were constructed by double-crossover recombination of a kanamycin-resistance cassette into the genome as previously described (Tian et al., 2007; Xu et al., 2007). The strategy for disrupting $d r 0091$ is shown in Supplementary Fig. S2(a). The 636 bp upstream and 537 bp downstream fragments of the dr0091 gene were amplified by the primers M91 P1/M91 P2

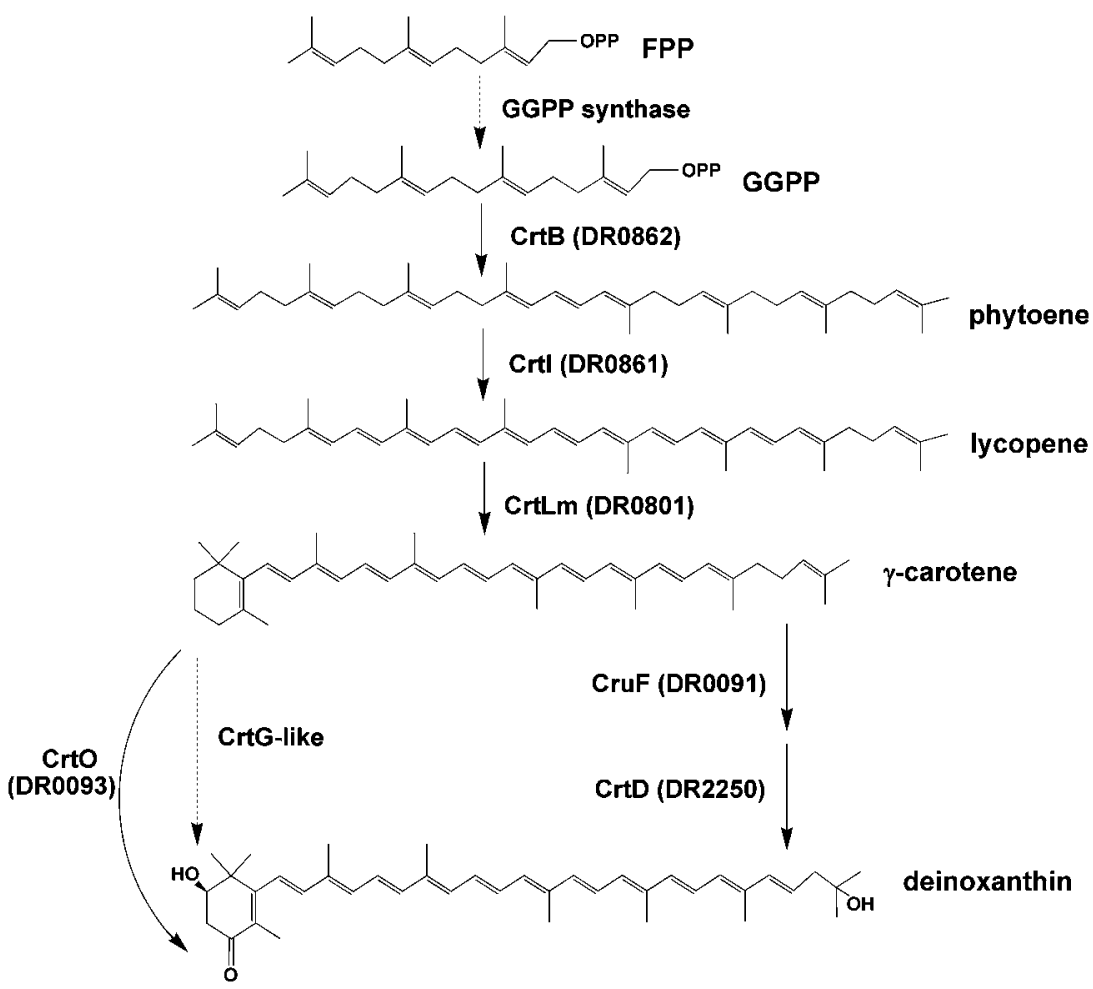

Fig. 1. Proposed main steps in the deinoxanthin biosynthetic pathway of $D$. radiodurans. Bold arrows refer to enzymes that have been experimentally confirmed in $D$. radiodurans R1 (CrtB, Crtl, CrtLm, CrtO, CrtD, CruF). Dotted arrows (CrtG-like, GGPP synthase) indicate hypothetical steps catalysed by enzymes that have not yet been identified in this bacterium. FPP, farnesyl diphosphate. 
Table 1. Bacterial strains and plasmids used in this study

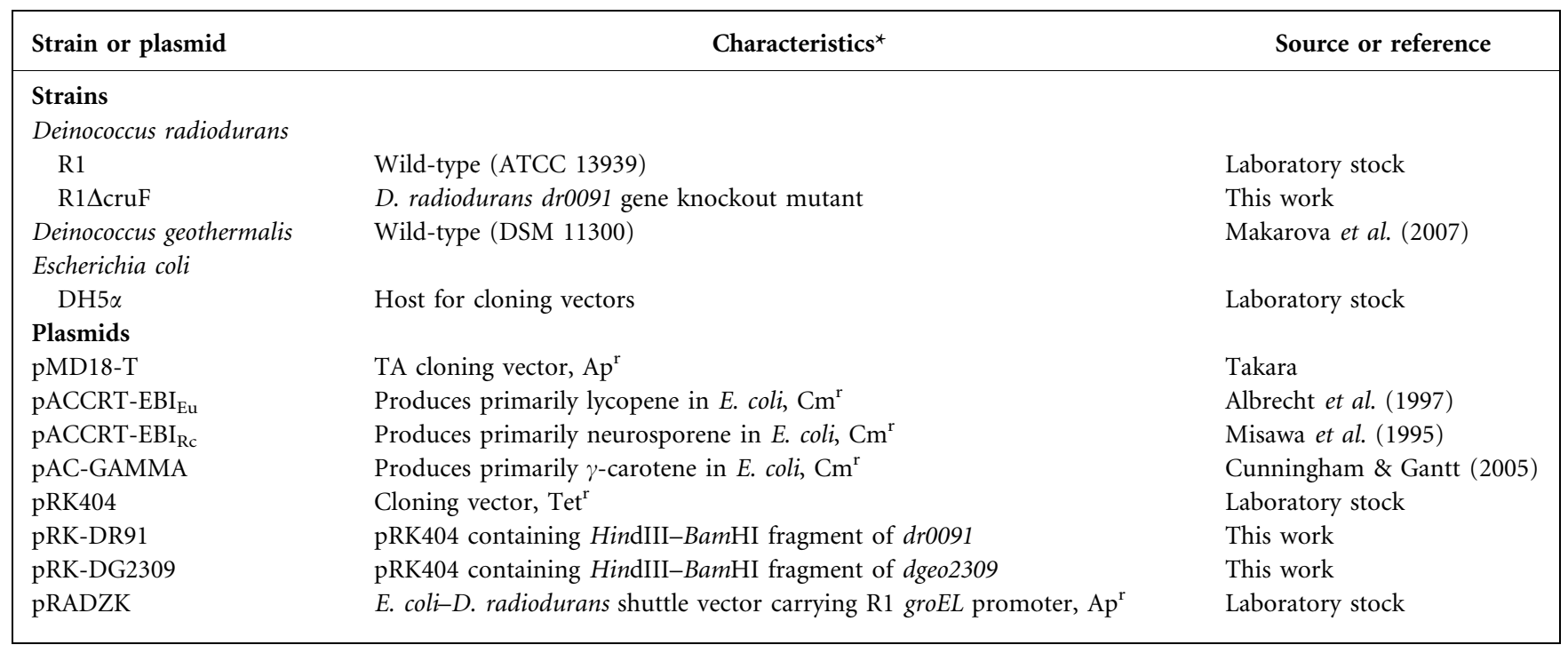

${ }^{\star} \mathrm{Ap}^{\mathrm{r}}$, ampicillin resistance; $\mathrm{Cm}^{\mathrm{r}}$, chloramphenicol resistance; $\mathrm{Tet}^{\mathrm{r}}$, tetracycline resistance.

(containing a HindIII restriction site) and M91 P3 (containing a BamHI restriction site)/M91 P4, respectively (Supplementary Table S1). The two fragments, digested by HindIII and BamHI, respectively, were ligated to the HindIII/BamHI-digested kanamycin gene. The joint product was transformed into $D$. radiodurans $\mathrm{R} 1$ by the $\mathrm{CaCl}_{2}$ method. The mutant strain, in which the DR0091 gene was replaced with a kanamycin-resistance gene, was plated and screened out on TGY agar containing kanamycin, and designated $\mathrm{R} 1 \Delta$ cruF. Gene mutation was confirmed by PCR product size analysis (Supplementary Fig. S2b). Furthermore, DNA sequencing of the PCR fragment from the mutant confirmed that the DR0091 gene had been replaced with a kanamycin-resistance cassette in the mutant.

Isolation and analysis of carotenoids. The method used to isolate the carotenoids was as described previously (Xu et al., 2007). A $100 \mathrm{ml}$ sample of each culture $\left(\mathrm{OD}_{600} 1.0\right)$ grown under aerobic conditions with continuous shaking was harvested by centrifugation at $5000 \mathrm{~g}$ for $10 \mathrm{~min}$. After washing three times with sterile water, the cell pellets were extracted three times with cold acetone/ methanol $(7: 2, \mathrm{v} / \mathrm{v})$ in the dark. The pooled carotenoid extracts were directly analysed by HPLC using a Waters 2690 Alliance system as previously described (Tian et al., 2008). A Hypersil ODS-C18 column $(4.6 \times 250 \mathrm{~mm}, 5 \mu \mathrm{m})$ was used and eluted with acetonitrile/ methanol/2-propanol $(40: 50: 10$, by vol.) at a flow rate of $1 \mathrm{ml}$ $\min ^{-1}$. The separated carotenoids were detected with a Waters 996 photodiode array detector and their absorption spectra were recorded online. The carotenoids were identified by their retention times and absorption spectral features, and by comparison with references (Saito et al., 1998; Steiger et al., 2003; Takaichi \& Shimada, 1992). LC-mass spectra of the carotenoids were recorded on an Agilent 1100 series LC/MSD Trap SL mass spectrometer system using atmospheric pressure chemical ionization (APCI). The system was controlled and data were analysed on a computer equipped with LC/MSD Trap Software 4.2 (Bruker). Detection was carried out in the negative ion mode with a corona current of $4.0 \mu \mathrm{A}$, a capillary voltage of $2.6 \mathrm{kV}$, a capillary exit voltage of $-129.6 \mathrm{~V}$, a dry temperature of $350{ }^{\circ} \mathrm{C}$, a vaporizer temperature of $425{ }^{\circ} \mathrm{C}$, high-purity $(99.999 \%)$ dry nitrogen gas at $8.01 \mathrm{~min}^{-1}$, and a nitrogen nebulizer pressure of $414 \mathrm{kPa}$.

\section{RESULTS}

\section{Identification of candidate genes}

Although a gene homologue of known CrtCs was not found in the D. radiodurans genome, it seemed likely that a carotenoid 1,2-hydratase of different sequence character would be present in the genome. A CrtC homologue was also not found in the genome sequence of $D$. geothermalis (Makarova et al., 2007). By comparative genomic analysis, the homologues of CrtB, CrtI, CrtLm, CrtO and CrtD in D. geothermalis were predicted to be Dego0523, Dgeo0524, Dgeo0857, Dgeo2310 and Dgeo2306, which have 65\%, $79 \%, 61 \%, 84 \%$ and $73 \%$ identity to those of $D$. radiodurans, respectively. Since carotenoid synthesis genes in some bacteria are assembled in clusters or in neighbourhoods to carry out their functions (Giraud et al., 2004; To et al., 1994), the gene neighbourhood of $\mathrm{crtO}$ in the genome D. radiodurans $\mathrm{R} 1$ and D. geothermalis DSM 11300 was inspected for gene candidates encoding carotenoid biosynthetic enzymes (Supplementary Fig. S1). We identified two ORFs of unknown function (dr0091 and dgeo2309) in the neighbourhoods of $c r t O$ (dr0093) of $D$. radiodurans and the crtO homologue (dgeo2310) of $D$. geothermalis, respectively. Since the ORF dr0092 was predicted to encode a MuT family protein of $D$. radiodurans, it was not selected as the possible crt gene candidate. dr0091 and dgeo2309 were selected for functional analysis.

\section{Expression of dr0091 and dge02309 genes in $E$. coli producing neurosporene or lycopene}

To characterize $d r 0091$ and dgeo2309, the expression vector pRK-DR91 or pRK-DG2309 was co-transformed with 

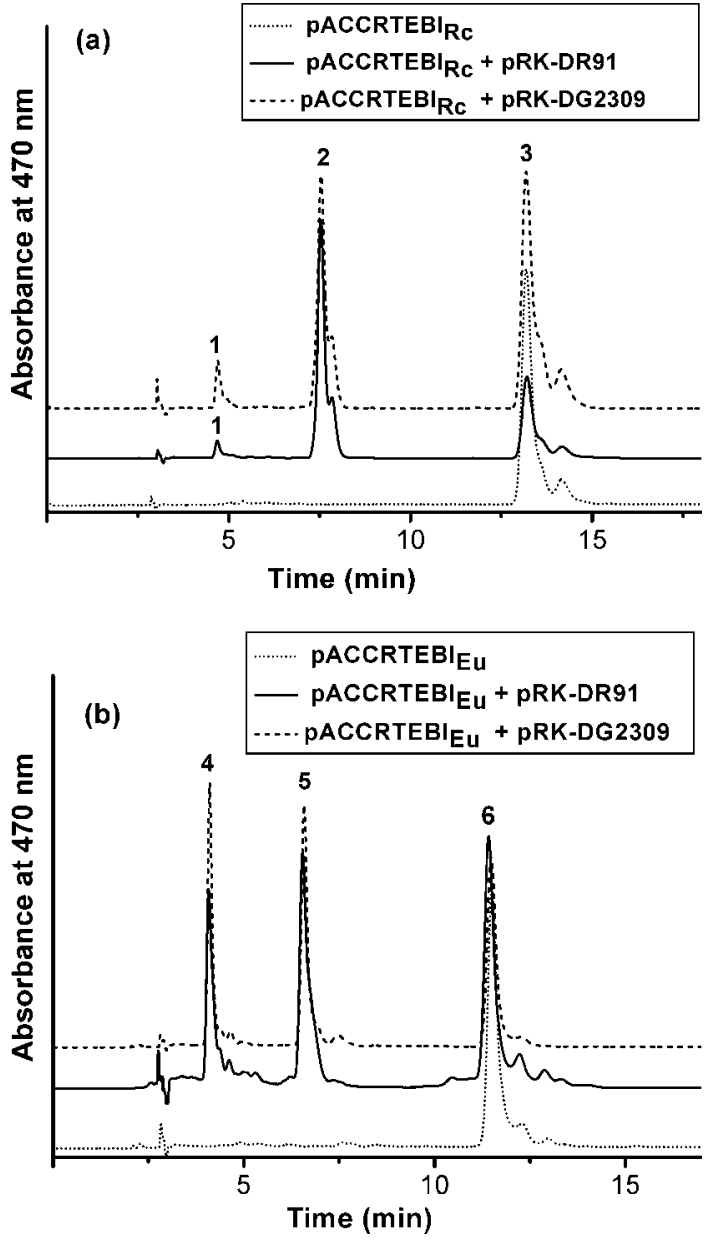

Fig. 2. Expression of dr0091 or dgeo2309 in neurosporeneproducing or lycopene-producing strains of $E$. coli. (a) HPLC analysis of carotenoids in neurosporene-producing $E$. coli transformants containing the plasmids pACCRT-EBI $\left.\right|_{R c}$, pACCRT-EBI $I_{\mathrm{Rc}}+\mathrm{pRK}-\mathrm{DR} 91$ or pACCRT-EBI $\mathrm{Rc}_{\mathrm{c}}+\mathrm{pRK}-\mathrm{DG} 2309$. (b) HPLC analysis of carotenoids in lycopene-producing $E$. coli transformants containing the plasmids pACCRT-EBI $E$, PACCRT$\mathrm{EBI}_{\mathrm{Eu}}+\mathrm{pRK}-\mathrm{DR} 91$ or pACCRT-EBI $\mathrm{Eu}+\mathrm{pRK}-\mathrm{DG} 2309$. Carotenoids were analysed by reverse-phase HPLC: column, Hypersil C18 (250×4.6 mm, $5 \mu \mathrm{m}$, Alltech); elution, acetonitrile/methanol/ 2-propanol ( $4: 5: 1$, by vol.); flow rate, $1 \mathrm{ml} \mathrm{min}^{-1}$; detection at $470 \mathrm{~nm}$. Peak 1, 1,1'-(OH)$)_{2}$-neurosporene; peak 2, 1-OHneurosporene; peak 3, neurosporene; peak 4, 1,1'-(OH)$)_{2}$-lycopene; peak 5, 1-OH-lycopene; peak 6, lycopene.

plasmid pACCRT-EBI $\mathrm{Rc}_{\mathrm{Rc}}$ or pACCRT-EBI $\mathrm{Eu}_{\mathrm{Eu}}$, respectively, into E. coli $\mathrm{DH} 5 \alpha$, which does not produce carotenoids. Carotenoid compositions in E. coli transformants were analysed by HPLC and LC-MS (Fig. 2, Table 2). E. coli containing pACCRT-EBI $\mathrm{Rc}_{\mathrm{Rc}}$ produced only neurosporene (peak 3 in Fig. 2a). When the dr0091 and dgeo2309 genes were expressed in the neurosporene-producing strain of $E$. coli, two other carotenoid products were observed (peaks 1 and 2 in Fig. 2a). The shorter retention times of peak 1 and peak 2 indicated that these products were more hydrophilic than neurosporene. Based on the retention time, absorbance spectrum and molecular mass of the products (Table 2), peak 1 ( $\lambda_{\max } 414,439,468 \mathrm{~nm}$ in HPLC mobile phase) with a mass of $574 \mathrm{Da}$ and peak $2\left(\lambda_{\max } 414,439,468 \mathrm{~nm}\right)$ with a mass of $556 \mathrm{Da}$ were identified as $1,1^{\prime}-(\mathrm{OH})_{2}$ neurosporene and 1-OH-neurosporene, respectively (Steiger et al., 2003). E. coli containing pACCRT-EBI produced only lycopene (peak 6 in Fig. 2b). Expression of the dr0091 and dgeo2309 genes in the lycopene-producing strain of $E$. coli resulted in the appearance of peak $4\left(\lambda_{\max }\right.$ $445,470,502 \mathrm{~nm})$ with a mass of $572 \mathrm{Da}$ and peak $5\left(\lambda_{\max }\right.$ $445,471,502 \mathrm{~nm}$ ) with a mass of $554 \mathrm{Da}$ (Fig. 2b; Table 2). Peaks 4 and 5 were identified as $1,1^{\prime}-(\mathrm{OH})_{2}$-lycopene and 1-OH-lycopene (Steiger et al., 2003), respectively. These results demonstrated that $d r 0091$ and dgeo2309 encode a carotenoid 1,2-hydratase that can not only convert neurosporene or lycopene into 1-OH-neurosporene or 1$\mathrm{OH}-$ lycopene, but also convert the monohydroxylated carotenoids 1-OH-neurosporene or 1-OH-lycopene into $1,1^{\prime}$ - $(\mathrm{OH})_{2}$-neurosporene or $1,1^{\prime}-(\mathrm{OH})_{2}$-lycopene.

\section{Expression of dr0091 and dge02309 genes in E. coli producing $\gamma$-carotene}

To further analyse the substrate specificity of the dr0091 and dgeo2309 gene products, the expression vector pRKDR91 or pRK-DG2309 was co-transformed with the plasmid pAC-GAMMA into E. coli DH5 $\alpha$. E. coli containing pAC-GAMMA produced $\gamma$-carotene (peak 8 in Fig. 3 ) and $\beta$-carotene (peak 9 in Fig. 3). Expression of $d r 0091$ and dgeo2309 in E. coli containing pAC-GAMMA resulted in the generation of a more hydrophilic product, peak 7 (8 min; $\lambda_{\max } 461,490 \mathrm{~nm}$; $554 \mathrm{Da}$ ) (Fig. 3 and Table 2). Based on the retention time, absorbance spectrum and molecular mass, peak 7 was identified as $1^{\prime}-\mathrm{OH}-\gamma$-carotene (Frigaard et al., 2004). These results demonstrated that DR0091 and Dgeo2309 can also convert monocyclic $\gamma$ carotene into $1^{\prime}-\mathrm{OH}-\gamma$-carotene.

\section{Mutant construction and carotenoid composition analysis of the mutant}

The strategy for disrupting $d r 0091$ is shown in Supplementary Fig. S2(a). Mutation of dr0091 resulted in a pink strain, designated $\mathrm{R} 1 \Delta \mathrm{cruF}$. The carotenoid composition of the mutant was analysed by HPLC and compared with the wild-type R1. As shown in Fig. 4(a), peak $1^{\prime}$ in mutant R1 $\Delta$ cruF had a retention time of $5.3 \mathrm{~min}$ and a $\lambda_{\max }$ of $468 \mathrm{~nm}$ and $490 \mathrm{~nm}$ in the mobile phase (Fig. 4c), while deinoxanthin (peak 1 in wild-type R1) had a shorter retention time, and a $\lambda_{\max }$ of $479 \mathrm{~nm}$ and $507 \mathrm{~nm}$ (Fig. 4b). The longer retention time and the spectral absorption blue shift of peak $1^{\prime}$ relative to deinoxanthin indicated that peak 1' was less hydrophilic and had fewer conjugated bonds (Takaichi \& Shimada, 1992; Takaichi, 2000). The absorbance spectrum of peak $1^{\prime}$ was similar to $3^{\prime}, 4^{\prime}$-dihydrodeinoxanthin $\left(\mathrm{C}_{40} \mathrm{H}_{56} \mathrm{O}_{3}\right)$, the major product of the mutant of carotenoid $3^{\prime}, 4^{\prime}$-desaturase (CrtD) (Tian 
Table 2. Carotenoid composition in E. coli transformants carrying various plasmids

\begin{tabular}{|c|c|c|c|c|}
\hline Plasmid & Carotenoids accumulated $^{*}$ & $\begin{array}{l}\text { Retention } \\
\text { time (min) }\end{array}$ & $\begin{array}{l}\text { Mol. mass } \\
\qquad(\mathrm{Da})\end{array}$ & $\begin{array}{c}\text { Spectroscopic properties } \\
{\left[\lambda_{\max }(\mathbf{n m}) \text { in HPLC eluent }\right] \dagger}\end{array}$ \\
\hline pACCRT-EBI ${ }_{\mathrm{Rc}}$ & Neurosporene & 13.2 & 538 & $416,440,468$ \\
\hline pACCRT-EBI ${ }_{R c}+p R K-D R 91$ & $\begin{array}{l}\text { 1-OH-neurosporene } \\
\text { Neurosporene }\end{array}$ & 7.3 & 556 & $414,439,468$ \\
\hline pACCRT-EBI ${ }_{\mathrm{Rc}}+$ pRK-DG2309 & $1,1^{\prime}-(\mathrm{OH})_{2}$-neurosporene & 4.4 & 574 & $414,439,468$ \\
\hline pACCRT-EBI ${ }_{\mathrm{Eu}}$ & Lycopene & 11.5 & 536 & $445,471,502$ \\
\hline \multirow[t]{2}{*}{ pACCRT-EBI $\mathrm{Eu}_{\mathrm{u}}+$ pRK-DR91 } & $1,1^{\prime}$-(OH $)_{2}$-lycopene & 4.1 & 572 & $445,470,502$ \\
\hline & $\begin{array}{l}\text { 1-OH-lycopene } \\
\text { Lycopene }\end{array}$ & 6.6 & 554 & $445,471,502$ \\
\hline pACCRT-EBI $\mathrm{Eu}_{1}+\mathrm{pRK}-\mathrm{DG} 2309$ & $1,1^{\prime}-(\mathrm{OH})_{2}$-lycopene & 4.1 & 572 & $445,470,502$ \\
\hline pAC-GAMMA & $\beta$-Carotene & 17.5 & 536 & $(424), 452,479$ \\
\hline pAC-GAMMA + pRK-DR91 & $1^{\prime}-\mathrm{OH}-\gamma$-carotene & 8.0 & 554 & $(435), 461,490$ \\
\hline pAC-GAMMA + pRK-DG2309 & $\begin{array}{l}\gamma \text {-Carotene, } \beta \text {-carotene } \\
1^{\prime} \text {-OH- } \gamma \text {-carotene } \\
\gamma \text {-Carotene, } \beta \text {-carotene }\end{array}$ & 8.0 & 554 & $(435), 461,490$ \\
\hline
\end{tabular}

${ }^{*}$ Carotenoids were analysed by reverse-phase HPLC: column, $5 \mu \mathrm{m}$ Hypersil C18 $(250 \times 4.6 \mathrm{~mm}$, Alltech); elution, acetonitrile/methanol/2propanol (4:5:1, by vol.); flow rate, $1 \mathrm{ml} \mathrm{min}^{-1}$; detection, $470 \mathrm{~nm}$.

†Wavelengths in parentheses indicate a shoulder rather than a distinct peak.

et al., 2008). From its main absorption maximum $\left(\lambda_{\max }\right.$ $468 \mathrm{~nm}$ in HPLC eluate), peak $1^{\prime}$ was suggestive of 12 conjugated double bonds including a conjugated keto group (Takaichi \& Shimada, 1992; Takaichi, 2000). Mass analysis showed peak $1^{\prime}$ with a mass of $566 \mathrm{Da}$ (matching

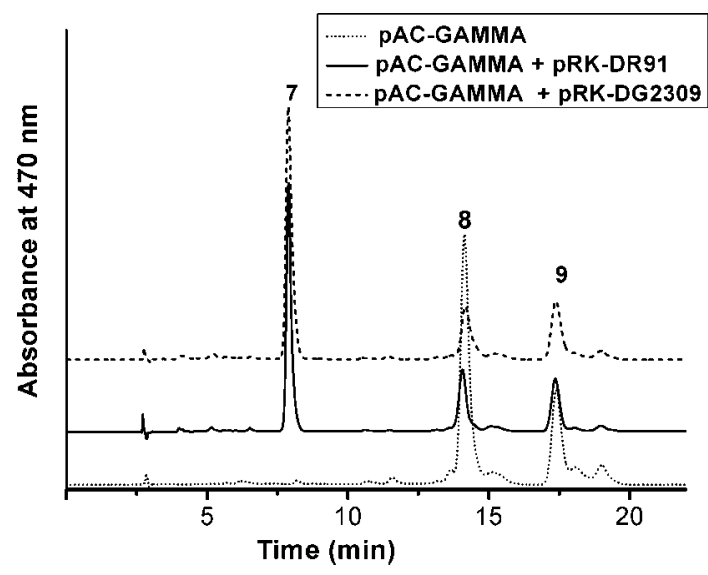

Fig. 3. Expression of the dr0091 or dgeo2309 in a $\gamma$-caroteneproducing strain of E. coli: HPLC analysis of carotenoids in E. coli transformants containing the plasmids pAC-GAMMA, pACGAMMA + pRK-DR91 or pAC-GAMMA + pRK-DG2309. Peak 7, $1^{\prime}$-OH- $\gamma$-carotene; peak $8, \gamma$-carotene; peak $9, \beta$-carotene. the formula $\mathrm{C}_{40} \mathrm{H}_{54} \mathrm{O}_{2}$ ). The spectral properties and mass result suggested that peak $1^{\prime}$ was 2-hydroxy-4-keto- $\gamma$ carotene, which is related to $3^{\prime}, 4^{\prime}$-dihydrodeinoxanthin but lacks a hydration modification. Our previous study showed that CrtD (DR2250) in D. radiodurans cannot catalyse C$3^{\prime}, 4^{\prime}$ desaturation of $\gamma$-carotene derivatives without the hydration reaction at the $\mathrm{C}-1^{\prime}, 2^{\prime}$ double bond (Tian et al., 2008). Therefore, DR0091 was confirmed to be a carotenoid 1,2-hydratase, which catalyses the hydration reaction before the $\mathrm{C}-3^{\prime}, 4^{\prime}$ desaturation step by CrtD (Fig. 1).

Peak $2^{\prime}$ in mutant $\mathrm{R} 1 \Delta \mathrm{cruF}$ had a retention time of $7.25 \mathrm{~min}$ with a $\lambda_{\max }$ of $468 \mathrm{~nm}$ and $486 \mathrm{~nm}$ in the mobile phase (Fig. $4 d$ ). Peak 2' showed a mass of $550 \mathrm{Da}$ (matching the formula $\mathrm{C}_{40} \mathrm{H}_{54} \mathrm{O}$ ). Based on the absorbance spectrum, mass and reference (Sandmann et al., 2006), peak $2^{\prime}$ was identified as 4 -keto- $\gamma$-carotene. Peak $3^{\prime}$ showed the same retention time (14.2 $\mathrm{min})$ and absorbance spectrum ( $\lambda_{\max } 461$, $491 \mathrm{~nm}$ ) (Fig. $4 \mathrm{e}$ ) as the reference $\gamma$-carotene (Table 2; peak 8 in Fig. 3); therefore, peak $3^{\prime}$ was identified as $\gamma$-carotene. Lycopene or earlier intermediates were not detected in the mutant strain, suggesting that DR0091 cannot catalyse these acyclic substrates in the native host cell.

\section{Phylogenetic analysis of carotenoid 1,2-hydratase}

Sequences of DR0091 homologues were retrieved using the BLAST program (http://www.ncbi.nlm.nih.gov/). DR0091 

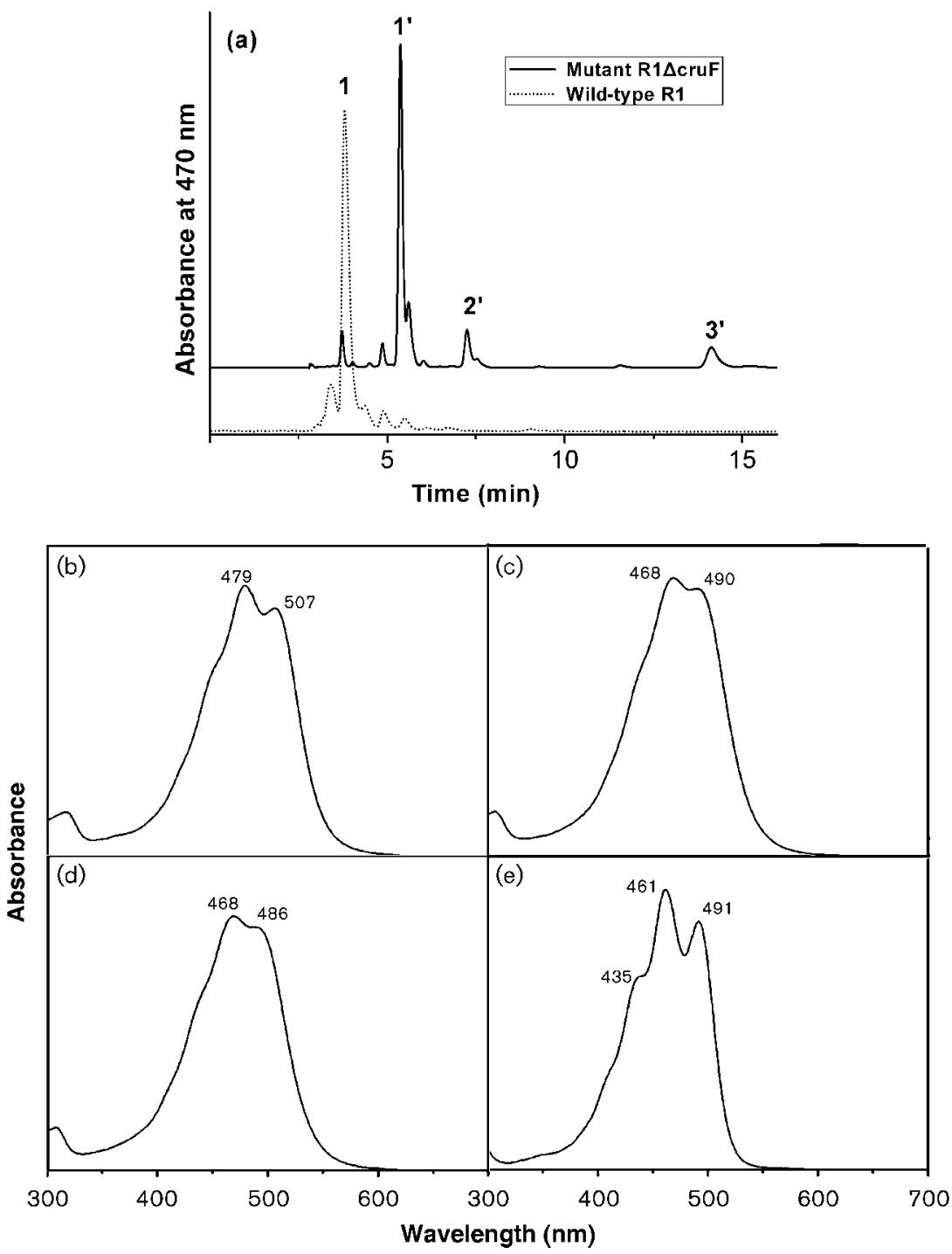

Fig. 4. Analysis of carotenoids from wild-type R1 and mutant R1 $\Delta$ cruF. (a) HPLC elution profile of carotenoids from wild-type R1 and mutant R1 $\Delta$ cruF. (b-e) Absorption spectra of identified peaks. Peak 1, deinoxanthin (b); peak 1', 2-hydroxy-4-keto- $\gamma$-carotene (c); peak 2', 4-keto- $\gamma$-carotene (d); peak $3^{\prime}, \gamma$ carotene (e). showed $63 \%$ identity to Dgeo2309. Their homologues found in carotenoid-producing bacteria were present in the DUF422 superfamily. The DR0091 amino acid sequence was found to be $35 \%$ identical to that of carotenoid hydratase (CruF) from Synechococcus sp. PCC 7002 (Maresca et al., 2008). Phylogenetic analysis showed that DR0091, Dgeo2309 and other CruF homologues were closer to each other than to CrtC homologues (Fig. 5). DR0091 and Dgeo2309 together with other CruF homologues formed a separate family distant from the known CrtC family.

\section{DISCUSSION}

Carotenoid 1,2-hydratase, a carotenoid biosynthetic enzyme, is required to catalyse the synthesis of deinoxanthin by hydration at C-1' $2^{\prime}$. In the present study, we identified two ORFs of previously unknown function (dr0091 and dgeo2309) as gene candidates of carotenoid 1,2-hydratase in the neighbourhoods of crtO of $D$. radiodurans and the crtO homologue of $D$. geothermalis, respectively. Combining gene expression and disruption analysis, we confirmed that $d r 0091$ and dgeo2309 encode a novel carotenoid 1,2-hydratase in D. radiodurans and $D$. geothermalis. The genes dr0091 and dgeo2309 showed little homology to the CrtC-type carotenoid 1,2-hydratase, which is found mainly in photosynthetic bacteria (Armstrong et al., 1989; Frigaard et al., 2004; Giraud et al., 2004; Kovács et al., 2003; Lang et al., 1995; Ouchane et al., 1997; Scolnik et al., 1980) and in the non-photosynthetic bacterium Myxococcus xanthus (Botella et al., 1995). Recently, a carotenoid 1,2-hydratase homologue of DR0091 and Dgeo2309 from the cyanobacterium Synechococcus sp. PCC 7002 was described and named CruF in a review (Maresca et al., 2008). This enzyme was shown to be required for the first committed step in myxoxanthophyll synthesis, and the hydration catalysed by the CruF from Synechococcus sp. PCC 7002 could be introduced both on acyclic lycopene and on monocyclic 


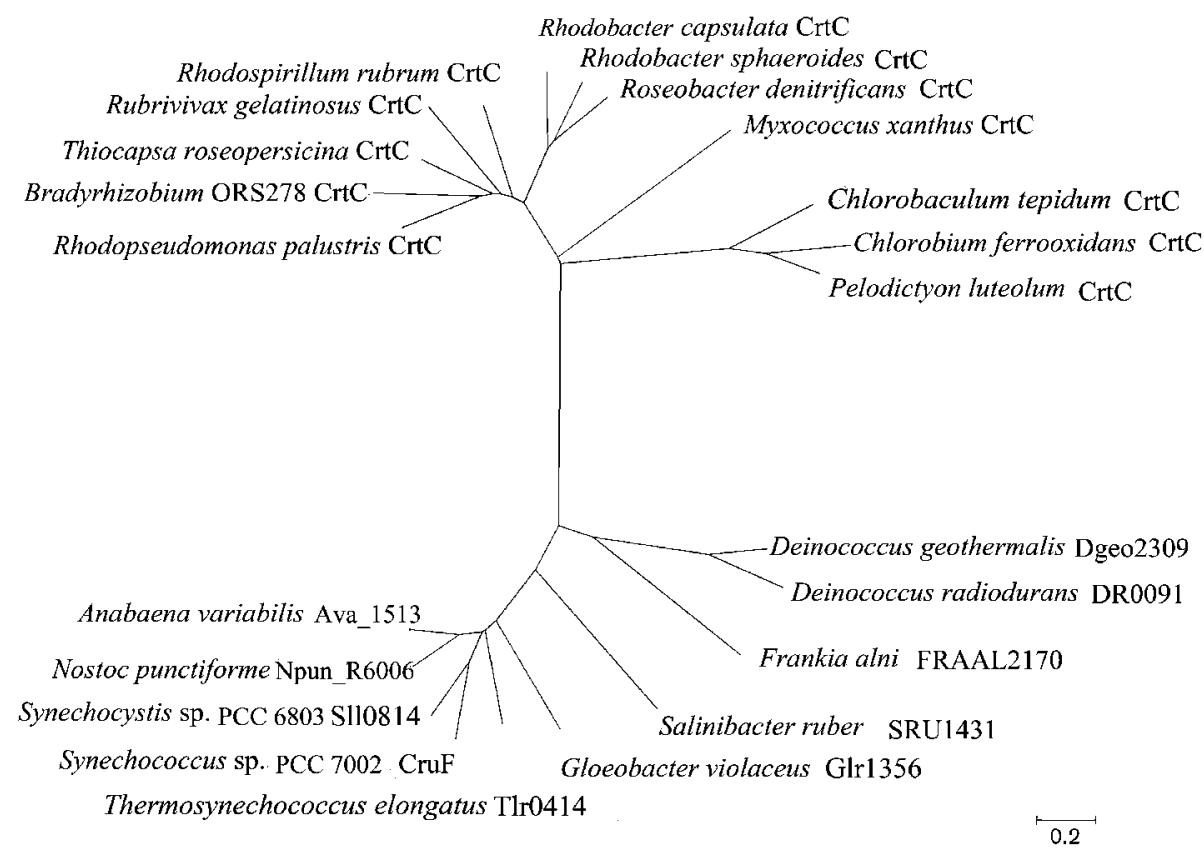

Fig. 5. Neighbour-joining phylogenetic tree of carotenoid 1,2-hydratases. Sequences analysed: Deinococcus radiodurans R1 DR0091 (NP_293817.1), Deinococcus geothermalis DSM 11300 Dgeo2309 (YP_605770.1), Synechococcus sp. PCC 7002 CruF (YP_001735274.1), Gloeobacter violaceus PCC 7421 Glr1356 (NP_924302.1), Thermosynechococcus elongatus BP-1 Tlr0414 (NP_681204.1), Anabaena variabilis ATCC 29413 Ava_1513 (YP_322031.1), Synechocystis sp. PCC 6803 SII0814 (NP_441438.1), Nostoc punctiforme PCC 73102 Npun_R6006 (YP_001869239.1), Salinibacter ruber DSM 13855 SRU1431 (YP_445555.1), Frankia alni ACN14a FRAAL2170 (YP_712398.1), Myxococcus xanthus DK1050 CrtC (CAA79959.1), Pelodictyon luteolum DSM 273 CrtC (Q3B257), Chlorobium ferrooxidans DSM 13031 CrtC (Q0YRY6), Chlorobaculum tepidum TLS CrtC (NP_661205.1), Roseobacter denitrificans OCh114 CrtC (Q16DU2), Rhodobacter sphaeroides 2.4.1 CrtC (YP_353342.1), Rhodobacter capsulatus CrtC (P17058), Rhodospirillum rubrum S1 CrtC (AAN75036.1), Thiocapsa roseopersicina BBS CrtC (Q7X3G5), Rubrivivax gelatinosus S1 CrtC (P95619), Bradyrhizobium sp. ORS278 CrtC (YP_001203729.1) and Rhodopseudomonas palustris CGA009 CrtC (NP_946865.1).

$\gamma$-carotene (Graham \& Bryant, 2009). The authors reported that homologues of CruF were also detected in the genomes of all sequenced cyanobacterial strains that produce myxol or the acylic xanthophyll oscillaxanthin. Cyclization of lycopene by lycopene cyclase may be either before or after the hydroxylation step catalysed by the CruF from Synechococcus sp. PCC 7002. However, in our analysis of the carotenoid intermediates in the mutant $\mathrm{R} 1 \Delta \mathrm{cruF}$, no acyclic lycopene derivatives or earlier intermediates were detected in the mutant, suggesting that DR0091 cannot catalyse the acyclic substrates in the native host. The CruF in $D$. radiodurans may catalyse the hydration reaction at the $\mathrm{C}-1^{\prime}, 2^{\prime}$ double bond of the monocyclic precursor of deinoxanthin. This result is consistent with our previous work on a mutant of lycopene cyclase (DR0801), in which no hydroxylated products of lycopene were detected, only lycopene, confirming that substituents on the end group including $\mathrm{C}-1^{\prime}, 2^{\prime}$ hydration and $\mathrm{C}-3^{\prime}, 4^{\prime}$ desaturation should be added after the cyclization of lycopene (Tian et al., 2008). To our knowledge, this is the first report of a functional carotenoid 1,2-hydratase (CruF) in non-photosynthetic bacteria. The identification of the CruF from $D$. geothermalis indicated that the major carotenoids might be hydroxylcarotenoids with C1 (or 1') hydroxyl groups. However, two major carotenoid products with retention times (13.5 $\mathrm{min}$ and $15.4 \mathrm{~min}$ ) different from deinoxanthin (3.4 min) were found in D. geothermalis using the same HPLC conditions (unpublished data). This indicated that the carotenoid composition and biosynthetic pathway of $D$. geothermalis may be different from that of D. radiodurans.

The functional analysis of DR0091 in the present study helped to reveal the deinoxanthin biosynthetic pathway in $D$. radiodurans. Disruption of the $d r 0091$ gene $(c r u F)$ not only blocked the hydration at $\mathrm{C}-1^{\prime}, 2^{\prime}$, but also inactivated the desaturation at $\mathrm{C}-3^{\prime}, 4^{\prime}$. This result was consistent with our previous findings that the $\mathrm{C}-3^{\prime}, 4^{\prime}$ desaturation was catalysed by CrtD (DR2250) following a hydration reaction at C-1',2' (Tian et al., 2008). Another carotenogenic enzyme in the proposed pathway not yet identified in $D$. radiodurans is 2-hydroxylase. A carotenoid $2,2^{\prime}$ - $\beta$-hydroxylase (CrtG) was detected in Brevundimonas sp. strain SD212 (Nishida et al., 2005). Recently, a CrtG was found in the carotenoid biosynthetic pathway of Thermosynechococcus elongatus strain $\mathrm{BP}-1$; this was the first functional identification of $\mathrm{CrtG}$ in a cyanobacterium (Iwai et al., 
2008). However, no homologues of this gene have been found in the genome of $D$. radiodurans.

The phylogenetic tree (Fig. 5) demonstrated the close evolutionary relationship among the CruF homologues. A similar close evolutionary relationship was also shown between the lycopene cyclase (CrtLm) from $D$. radiodurans and the CrtL from Synechococcus sp. PCC 7942 (Krubasik \& Sandmann, 2000). The CruF homologues formed a separate clade of carotenoid 1,2-hydratase, and are evolutionarily distant from the known hydroxyneurosporene synthase ( $\mathrm{CrtC})$ clade. Since cyanobacteria and nonphotosynthetic Deinococcus are grouped in the same cluster, it may be inferred that lateral gene transfer has occurred between these bacteria. It has been suggested that lateral gene transfer events may have made a substantial contribution to the distribution of genes for carotenoid biosynthesis across bacterial genomes (Phadwal, 2005).

The antioxidant capacity of carotenoids is thought to be linked to the length of their conjugated double bond system and the presence of functional groups (Albrecht et al., 2000). Besides an extension of the conjugated double bond, there is an additional hydroxyl group substitution at $\mathrm{C}-1^{\prime}$ of the double bonds in deinoxanthin. The presence of the hydroxyl group may enhance its scavenging activity by hydrogen abstraction reactions with ROS. Hydroxylycopene has been reported to have greater antioxidant properties than lycopene because of the presence of a hydroxyl group at position C-1' (Albrecht et al., 2000). Therefore, carotenoid 1,2-hydratase (DR0091), responsible for the hydration at C$1^{\prime}, 2^{\prime}$ in deinoxanthin biosynthesis, is important for the antioxidant activity of deinoxanthin. As non-enzymic ROS scavengers, carotenoids in $D$. radiodurans may act together with pyrroloquinoline quinone (Misra et al., 2004) and a high intracellular Mn(II) to Fe(II) ratio (Daly et al., 2007) in intracellular resistance against oxidative stress.

In this study, we identified and characterized a novel carotenoid 1,2-hydratase (CruF) in the non-photosynthetic bacteria $D$. radiodurans and $D$. goethermalis. We also determined the possible reaction sequences involved in deinoxanthin biosynthesis. The carotenoid biosynthetic enzymes identified from Deinococcus can also be used, via genetic engineering, for the production of novel carotenoids with high activities. Further investigations of the remaining unidentified carotenoid biosynthetic enzymes are needed to elucidate the carotenoid biosynthetic pathway in Deinococcus fully.

\section{ACKNOWLEDGEMENTS}

We thank Professor G. Sandmann for plasmids pACCRT-EBI $\mathrm{Rc}_{\mathrm{Rc}}$ and pACCRT-EBI ${ }_{\mathrm{Eu}}$, and Professor F. X. Cunningham, Jr, for plasmid pAC-GAMMA. This work was supported by grants from the National Basic Research Program of China (2004CB19604, 2007CB707804), a grant from the National Hi-Tech Development Program (2007AA021305), a key project from the National Natural Science Foundation of China (30830006), grants from the National Natural Science Foundation of China $(30670026,30870035)$, the project
'Application of Nuclear Techniques in Agriculture' from the Chinese Ministry of Agriculture (200803034), a project from Zhejiang Provincial Natural Science Foundation of China (Y306075) and a project from Science and Technology Department of Zhejiang Province of China (2006E10058).

\section{REFERENCES}

Albrecht, M., Takaichi, S., Misawa, N., Schnurr, G., Böger, P. \& Sandmann, G. (1997). Synthesis of atypical cyclic and acyclic hydroxy carotenoids in Escherichia coli transformants. J Biotechnol 58, 177-185.

Albrecht, M., Takaichi, S., Steiger, S., Wang, Z. Y. \& Sandmann, G. (2000). Novel hydroxycarotenoids with improved antioxidative properties produced by gene combination in Escherichia coli. Nat Biotechnol 18, 843-846.

Armstrong, G. A. (1997). Genetics of eubacterial carotenoid biosynthesis: a colorful tale. Annu Rev Microbiol 51, 629-659.

Armstrong, G. A., Alberti, M., Leach, F. \& Hearst, J. E. (1989). Nucleotide sequence, organization, and nature of the protein products of the carotenoid biosynthesis gene cluster of Rhodobacter capsulatus. Mol Gen Genet 216, 254-268.

Botella, J. A., Murillo, F. J. \& Ruiz-Vázquez, R. (1995). A cluster of structural and regulatory genes for light-induced carotenogenesis in Myxococcus xanthus. Eur J Biochem 233, 238-248.

Cox, M. M. \& Battista, J. R. (2005). Deinococcus radiodurans - the consummate survivor. Nat Rev Microbiol 3, 882-892.

Cunningham, F. X., Jr \& Gantt, E. (2005). A study in scarlet: enzymes of ketocarotenoid biosynthesis in the flowers of Adonis aestivalis. Plant J 41, 478-492.

Daly, M. J., Gaidamakova, E. K., Matrosova, V. Y., Vasilenko, A., Zhai, M., Leapman, R. D., Lai, B., Ravel, B., Li, S. M. \& other authors (2007). Protein oxidation implicated as the primary determinant of bacterial radioresistance. PLoS Biol 5, e92.

Ferreira, A. C., Nobre, M. F., Rainey, F. A., Silva, M. T., Wait, R., Burghardt, J., Chung, A. P. \& da Costa, M. S. (1997). Deinococcus geothermalis sp. nov. and Deinococcus murrayi sp. nov., two extremely radiation-resistant and slightly thermophilic species from hot springs. Int J Syst Bacteriol 47, 939-947.

Frigaard, N. U., Maresca, J. A., Yunker, C. E., Jones, A. D. \& Bryant, D. A. (2004). Genetic manipulation of carotenoid biosynthesis in the green sulfur bacterium Chlorobium tepidum. J Bacteriol 186, 5210-5220.

Giraud, E., Hannibal, L., Fardoux, J., Jaubert, M., Jourand, P., Dreyfus, B., Sturgis, J. N. \& Vermeglio, A. (2004). Two distinct crt gene clusters for two different functional classes of carotenoid in Bradyrhizobium. J Biol Chem 279, 15076-15083.

Graham, J. E. \& Bryant, D. A. (2009). The biosynthetic pathway for myxol-2' fucoside (myxoxanthophyll) in the cyanobacterium Synechococcus sp. strain PCC 7002. J Bacteriol 191, 3292-3300.

Iwai, M., Maoka, T., Ikeuchi, M. \& Takaichi, S. (2008). 2,2'- $\beta$ Hydroxylase $(\mathrm{CrtG})$ is involved in carotenogenesis of both nostoxanthin and 2-hydroxymyxol 2'-fucoside in Thermosynechococcus elongatus strain BP-1. Plant Cell Physiol 49, 1678.

Kovács, A. T., Rákhely, G. \& Kovács, K. L. (2003). Genes involved in the biosynthesis of photosynthetic pigments in the purple sulfur photosynthetic bacterium Thiocapsa roseopersicina. Appl Environ Microbiol 69, 3093-3102.

Krubasik, P. \& Sandmann, G. (2000). Molecular evolution of lycopene cyclases involved in the formation of carotenoids with ionone end groups. Biochem Soc Trans 28, 806-810.

Lang, H. P., Cogdell, R. J., Takaichi, S. \& Hunter, C. N. (1995). Complete DNA sequence, specific Tn5 insertion map, and gene 
assignment of the carotenoid biosynthesis pathway of Rhodobacter sphaeroides. J Bacteriol 177, 2064-2073.

Lemee, L., Peuchant, E., Clerc, M., Brunner, M. \& Pfander, H. (1997). Deinoxanthin: a new carotenoid isolated from Deinococcus radiodurans. Tetrahedron 53, 919-926.

Makarova, K. S., Aravind, L., Wolf, Y. I., Tatusov, R. L., Minton, K. W., Koonin, E. V. \& Daly, M. J. (2001). Genome of the extremely radiationresistant bacterium Deinococcus radiodurans viewed from the perspective of comparative genomics. Microbiol Mol Biol Rev 65, $44-79$.

Makarova, K. S., Omelchenko, M. V., Gaidamakova, E. K., Matrosova, V. Y., Vasilenko, A., Zhai, M., Lapidus, A., Copeland, A., Kim, E. \& other authors (2007). Deinococcus geothermalis: the pool of extreme radiation resistance genes shrinks. PLoS One 2, e955.

Maresca, J. A., Graham, J. E. \& Bryant, D. A. (2008). The biochemical basis for structural diversity in the carotenoids of chlorophototrophic bacteria. Photosynth Res 97, 121-140.

Misawa, N., Satomi, Y., Kondo, K., Yokoyama, A., Kajiwara, S., Saito, T., Ohtani, T. \& Miki, W. (1995). Structure and functional analysis of a marine bacterial carotenoid biosynthesis gene cluster and astaxanthin biosynthetic pathway proposed at the gene level. J Bacteriol 177, 65756584

Misra, H. S., Khairnar, N. P., Barik, A., Indira Priyadarsini, K., Mohan, H. \& Apte, S. K. (2004). Pyrroloquinoline-quinone: a reactive oxygen species scavenger in bacteria. FEBS Lett 578, 26-30.

Nishida, Y., Adachi, K., Kasai, H., Shizuri, Y., Shindo, K., Sawabe, A., Komemushi, S., Miki, W. \& Misawa, N. (2005). Elucidation of a carotenoid biosynthesis gene cluster encoding a novel enzyme, $2,2^{\prime}-\beta$ hydroxylase, from Brevundimonas sp. strain SD212 and combinatorial biosynthesis of new or rare xanthophylls. Appl Environ Microbiol 71, 4286-4296.

Ouchane, S., Picaud, M., Vernotte, C. \& Astier, C. (1997). Photooxidative stress stimulates illegitimate recombination and mutability in carotenoid-less mutants of Rubrivivax gelatinosus. EMBO J 16, 4777-4787.

Phadwal, K. (2005). Carotenoid biosynthetic pathway: molecular phylogenies and evolutionary behavior of crt genes in eubacteria. Gene 345, 35-43.

Saito, T., Ohyama, Y., Ide, H., Ohta, S. \& Yamamoto, O. (1998). A carotenoid pigment of the radioresistant bacterium Deinococcus radiodurans. Microbios 95, 79-90.

Sandmann, G., Zhu, C., Krubasik, P. \& Fraser, P. D. (2006). The biotechnological potential of the al-2 gene from Neurospora crassa for the production of monocyclic keto hydroxy carotenoids. Biochim Biophys Acta 1761, 1085-1092.

Schmidt-Dannert, C. (2000). Engineering novel carotenoids in microorganisms. Curr Opin Biotechnol 11, 255-261.
Scolnik, P. A., Walker, M. A. \& Marrs, B. L. (1980). Biosynthesis of carotenoids derived from neurosporene in Rhodopseudomonas capsulata. J Biol Chem 255, 2427-2432.

Steiger, S., Mazet, A. \& Sandmann, G. (2003). Heterologous expression, purification, and enzymatic characterization of the acyclic carotenoid 1,2-hydratase from Rubrivivax gelatinosus. Arch Biochem Biophys 414, 51-58.

Takaichi, S. (2000). Characterization of carotenes in a combination of a C18 HPLC column with isocratic elution and absorption spectra with a photodiode-array detector. Photosynth Res 65, 93-99.

Takaichi, S. \& Mochimaru, M. (2007). Carotenoids and carotenogenesis in cyanobacteria: unique ketocarotenoids and carotenoid glycosides. Cell Mol Life Sci 64, 2607-2619.

Takaichi, S. \& Shimada, K. (1992). Characterization of carotenoids in photosynthetic bacteria. Methods Enzymol 213, 374-385.

Tao, L. \& Cheng, Q. (2004). Novel $\beta$-carotene ketolases from nonphotosynthetic bacteria for canthaxanthin synthesis. Mol Genet Genomics 272, 530-537.

Tao, L., Picataggio, S., Rouviere, P. E. \& Cheng, Q. (2004). Asymmetrically acting lycopene $\beta$-cyclases (CrtLm) from nonphotosynthetic bacteria. Mol Genet Genomics 271, 180-188.

Tian, B., Xu, Z., Sun, Z., Lin, J. \& Hua, Y. (2007). Evaluation of the antioxidant effects of carotenoids from Deinococcus radiodurans through targeted mutagenesis, chemiluminescence, and DNA damage analyses. Biochim Biophys Acta 1770, 902-911.

Tian, B., Sun, Z., Xu, Z., Shen, S., Wang, H. \& Hua, Y. (2008). Carotenoid $3^{\prime}, 4^{\prime}$-desaturase is involved in carotenoid biosynthesis in the radioresistant bacterium Deinococcus radiodurans. Microbiology 154, 3697-3706.

To, K. Y., Lai, E. M., Lee, L. Y., Lin, T. P., Hung, C. H., Chen, C. L., Chang, Y. S. \& Liu, S. T. (1994). Analysis of the gene cluster encoding carotenoid biosynthesis in Erwinia herbicola Eho13. Microbiology 140, 331-339.

White, O., Eisen, J. A., Heidelberg, J. F., Hickey, E. K., Peterson, J. D., Dodson, R J., Haft, D. H., Gwinn, M. L., Nelson, W. C. \& other authors (1999). Genome sequence of the radioresistant bacterium Deinococcus radiodurans $\mathrm{R} 1$. Science 286, 1571-1577.

Xu, Z., Tian, B., Sun, Z., Lin, J. \& Hua, Y. (2007). Identification and functional analysis of a phytoene desaturase gene from the extremely radioresistant bacterium Deinococcus radiodurans. Microbiology 153, 1642-1652.

Zhang, L., Yang, Q., Luo, X., Fang, C., Zhang, Q. \& Tang, Y. (2007). Knockout of $c r t B$ or crtI gene blocks the carotenoid biosynthetic pathway in Deinococcus radiodurans $\mathrm{R} 1$ and influences its resistance to oxidative DNA-damaging agents due to change of free radicals scavenging ability. Arch Microbiol 188, 411-419.

Edited by: J. Green 